\title{
Factors that Influence the Development of Compassion Fatigue, Burnout, and Compassion Satisfaction in Emergency Department Nurses
}

\author{
Stacie Hunsaker \\ stacie-hunsaker@byu.edu \\ Dale Maughan \\ Sondra Heaston \\ sondra-heaston@byu.edu
}

Follow this and additional works at: https://scholarsarchive.byu.edu/facpub

Part of the Nursing Commons

\section{Original Publication Citation}

Journal of Nursing Scholarship 2014

\section{BYU ScholarsArchive Citation}

Hunsaker, Stacie; Maughan, Dale; and Heaston, Sondra, "Factors that Influence the Development of Compassion Fatigue, Burnout, and Compassion Satisfaction in Emergency Department Nurses" (2014). Faculty Publications. 1549.

https://scholarsarchive.byu.edu/facpub/1549 


\begin{abstract}
Purpose: The purpose of this study was twofold: (1) to determine the prevalence of compassion satisfaction (CS), compassion fatigue (CF), and burnout in ED nurses throughout the United States and (2) to determine which demographic and work-related components affect the development of CS, CF, and burnout in this nursing specialty.

Design/Methods: This was a non-experimental, descriptive, and predictive study using a selfadministered survey. Survey packets including a demographic questionnaire and the Professional Quality of Life Scale version 5 (ProQOL 5) were mailed to 1,000 selected emergency nurses throughout the United States. The ProQOL 5 scale was used to measure the prevalence of CS, CF, and burnout among ED nurses. Multiple regression using stepwise solution was employed to determine which variables of demographics and work related characteristics predicted the prevalence of CS, CF, and burnout. The alpha level was set at .05 for statistical significance.
\end{abstract}

Findings: The results revealed overall low to average levels of CF and burnout and generally average to high levels of CS among this group of ED nurses. The low level of manager support was a significant predictor of higher levels of burnout and CF among ED nurses, while a high level of manager support contributed a higher level of CS.

Conclusion: The results may serve to help distinguish elements in ED nurses' work and life that are related to CS and may identify factors associated with higher levels of CF and burnout.

Clinical Relevance: Improving recognition and awareness of CS, CF, and burnout among ED nurses may prevent emotional exhaustion and help identify interventions that will help nurses remain empathetic and compassionate professionals.

Key Words: compassion fatigue, compassion satisfaction, burnout, emergency nurses 


\section{Introduction}

The profession of emergency nursing is physically and emotionally demanding.

Complex patient loads, long shifts, demanding physicians, a fast-paced environment, and working in an emotionally and physically challenging area can cause stress for emergency department (ED) nurses (Hooper, Craig, Janvrin, Wetsel, \& Reimels, 2010; Von Rueden et al. 2010; Healy \& Tyrrell 2011). Compassion fatigue (CF) and burnout are conditions which can become overwhelming burdens on nurses and can cause physical, mental, and emotional health difficulties (Potter, 2006). A nurse affected by CF may become ineffective, depressed, apathetic, and detached (Boyle, 2011). CF and nurse burnout negatively affect both the caregiver and the patient. Moreover, high levels of nurse burnout have been linked to patient dissatisfaction (Vahey, Aiken, Sloane, Clarke, \& Vargas, 2004). Therefore, it is imperative that CF and burnout be recognized and addressed. By studying the impact of CF and burnout on ED nurses, researchers may bring to the attention of managers, healthcare leaders, and nurses themselves, the reality of this phenomenon and aid in the comprehension of its negative influence.

Additionally, the complexity of patient care is climbing, resources are decreasing, and insurance reimbursement is being linked to patient satisfaction (Medicare, 2013). It is more important now, perhaps more than any other time in healthcare, to understand the prevalence and predictors of $\mathrm{CF}$ and burnout, but also compassion satisfaction (CS) in ED nurses. By understanding factors that influence both positive and negative aspects of nurses' work, perhaps levels of awareness will be raised and nurses may maintain caring relationships and positive attitudes. However, few studies were conducted to explore factors that influence the prevalence of CF and burnout on ED nurses (Dominguez-Gomez \& Rutledge, 2009; Hooper et al., 2010). Thus, the purpose of this study was to determine the prevalence of CS, CF, and burnout in ED 
nurses throughout the United States and to determine which demographic and work-related components affect the development of CS, CF, and burnout in this nursing specialty

Based on the purpose of the study, the research questions were: (1) What is the prevalence of CS, CF, and burnout among ED nurses? (2) What demographic characteristics such as age and gender are associated with the prevalence of CS, CF, and burnout among ED nurses? (3) What work-related characteristics such as educational level, years in nursing, shift length, years worked in the ED, hours worked per week, and having adequate manager support are significantly associated with the prevalence of CS, CF, and burnout among ED nurses? (4) To what extent do the variables of demographics and work-related characteristics predict the prevalence of having CS, CF, and burnout among ED nurses, respectively?

\section{Literature Review}

The term compassion fatigue was first introduced by Joinson in 1992. She described CF as nurses losing their ability to nurture. Compassion fatigue has been defined as the negative consequences of working with a significant number of traumatized individuals in combination with a strong, personal, empathic orientation. Figley (1995), a noted early researcher on CF commented that those who are in a caring profession have an enormous capacity for feeling and expressing empathy tend to be more at risk for CF. Humans, by nature, are wired for empathy, and therefore, caregiving can take a toll both emotionally and physically (Flarity, 2011).The stress resulting from helping a traumatized or suffering person may result in $\mathrm{CF}$, which develops as a self-protection measure (Figley, 1995).

While CF is caused by empathy, burnout is associated with environmental factors such as high patient acuity, overcrowding, and problems with administration (Flarity, 2013). Burnout is a condition often associated with feelings of hopelessness and inability to perform job duties 
effectively (Stamm, 2010). Burnout and CF are often linked and closely mimic one another. CF is often described as a type of burnout (Portnoy, 2011). A principle difference between burnout and CF is burnout typically exhibits a gradual onset while CF may occur suddenly. Although measuring negative aspects of a nurse's job is important; it is equally valuable to determine what makes a nurse feel happy. Compassion satisfaction is the positive aspect of helping others. It is the satisfaction achieved with one's work by helping others and being able to do one's job well (Stamm, 2010). Many nurses chose their profession specifically to help others.

$\mathrm{CF}$ and burnout may have severe professional consequences in addition to affecting a nurse's personal well-being. CF and burnout affect nurse retention, patient safety, and patient satisfaction (Burtson \& Stichler, 2010; Potter et al., 2010). Hospitals are expected not only to provide positive outcomes for patients, but make them happy while providing quality care. A relatively new performance measure for hospitals is patient satisfaction. Since 2007, the passage of health reform legislation has increased focus on the importance of the patient experience (McHugh, Kutney-Lee, Cimiotti, Sloane, \& Aiken, 2011). Therefore, Medicare reimbursements to hospitals are now partially based on patient satisfaction measurements. Thirty percent of the incentive payments provided by Medicare to hospitals is based on approval scores of satisfaction (Medicare, 2013).

Nurses who are experiencing CF and burnout are too exhausted to provide levels of care that help patients feel satisfied (Boyle, 2011; McHugh et al., 2011). As aforementioned, CS is the positive aspect of helping others (Stamm, 2010). Many nurses choose this profession because they experience fulfillment in helping others. Thus, understanding the factors that contribute to $\mathrm{CF}$ and burnout may help ED nurses maintain their ability to experience work fulfillment and contribute to patient satisfaction. 


\section{Empirical Studies Related to the Study Problem}

The need to identify the level of CF in ED nurses was clear throughout the literature review. The conclusions in most research reviewed portrayed high levels of CF in healthcare workers and indicated the need for further research regarding CF and burnout among ED nurses. To the researchers' knowledge, there have been only two quantitative studies precisely targeting CF in ED nurses (Hooper et al., 2010; Dominguez-Gomez \& Rutledge, 2009). Both studies had a limitation of a small sample size and studied CF in ED nurses in two specific geographical locations: a hospital in the Southeast United States, and three hospitals in California, respectively.

Hooper et al. (2010) compared levels of CS, CF, and burnout among ED, intensive care unit, oncology, and nephrology nurses. The Professional Quality of Life (ProQOL) scale was used to examine a difference in the level of CF and burnout in nurses' working in these different specialty units. Though this exploratory, cross-sectional study did not show a significantly statistical difference in CF levels of the nurses among those specialty units, it did attest that ED nurses were at risk for less CS compared to the other types of nurses. This study also revealed a greater risk for burnout in ED nurses and a greater risk for CF in oncology nurses.

The Dominguez-Gomez and Rutledge's (2009) study focused on measuring the level of CF in ED nurses, using the Secondary Traumatic Stress (STS) tool. It was the first quantitative exploration of CF in ED nurses. The findings of the study demonstrated high levels of CF among the ED nurse respondents. High levels of CF in nurses may affect patient care and contribute to burnout. The study suggested further research aims at increasing the awareness of this phenomenon, as well as a recommendation for managers and organizations to be more aware of 
the problems of $\mathrm{CF}$ and burnout and support nurses, and when appropriate, urge them to seek counseling (Dominguez-Gomez \& Rutledge, 2009).

Understandably, EDs are often considered to be a stressful work environment. Multiple studies have revealed that workplace violence, death/ resuscitations of patients, caring for trauma victims, and stressful events which occur frequently in this setting, contribute to increased stress in ED workers (Healy \& Tyrrell, 2011; Von Rueden et al., 2010). ED nurses must deal with unpredictable events which may include death, violence, and overcrowding. However, little evidence has shown to identify factors that are associated with ED nurses' demographics and work-related characteristics contributing to their CF, CS, and burnout. By identifying factors that may predict $\mathrm{CF}$ and burnout, as well as recognizing factors that improve satisfaction at work, may be useful in retaining ED nurses and develop strategies to support them to provide excellent care without compromising their own health and happiness.

\section{Conceptual Framework}

A number of theoretical frameworks were applied to guide studies related to CS, CF, and burnout such as Maslow's theory of hierarchy of needs and Watson's theory of human caring (Burtson \& Stichler, 2010). A most significant theoretical model developed by Figley (2002) was the stress-process framework. This model was developed based on factors that contribute to CF. Figley discovered that CF develops as a result of a caregiver's exposure to his/her patients' experiences joined with his/her natural empathy. Later on, Stamm (2010) applied the CS-CF model to the development of the ProQOL scale. The CS-CF model illustrates a theoretical path analysis of positive outcomes (CS) and negative outcomes (CF) of helping those who have experienced traumatic stress.

Based on Stamm's (2010) theoretical path analysis diagram, a conceptual framework related to $\mathrm{CS}, \mathrm{CF}$, and burnout among ED nurses was developed to guide this study. The researchers 
believe that individual and organizational characteristics may contribute to and have an influence on the development of CS, CF, and burnout. Several variables were identified according to literature reviews. The demographic independent variables were age and gender. The workrelated independent variables were level of education, years in profession, hours of work per week, length of shift, years as an ED nurse, and manager support. The dependent variables included CS, CF, and burnout.

\section{Methods}

\section{Sample and Population}

This cross-sectional study used a non-experimental, descriptive, and predictive design. The target population for this study was registered nurses (RNs) who worked in emergency departments throughout the United States. The inclusion criteria for participation were: (1) Work at least 8 hours per week in the ED, (2) interact directly with ED patients at least 8 hours per week, and (3) have at least one year of experience in the ED. The rationale for including a minimum of at least one year experience in the ED and working at least 8 hours per week was the consideration of having experience and exposure frequently enough to traumatic events that contribute to the development of $\mathrm{CF}$ and burnout. According to a list of ED nurse members with mailing addresses throughout the United States provided by the Emergency Nurses Association (ENA), a purposive sampling was used to recruit the total 1,000 ED nurses in this study.

\section{Data Collection Procedure}

Approval from the Institutional Review Board (IRB) of the University was obtained prior to any data collection. The survey packet including a letter of explanation, an informed consent letter, a copy of the demographic questionnaire, and a copy of the ProQOL version 5 scale was mailed to each potential participant. The participants returned the surveys to the 
researchers in a provided self-addressed stamped envelope. In order to maximize the response rate, two follow-up postcard reminders were sent to all 1,000 potential participants at 2-week and 6-week intervals, respectively, from the original survey mailing date. The researchers took every precaution possible to protect the anonymity and privacy of the individuals. The survey was answered anonymously and kept confidential in reporting the results of the study by removing identifying information. To protect confidentiality, all data were numerically coded and accessible only by the researchers.

\section{Instrumentation}

The survey used in this study included the Professional Quality of Life Compassion Satisfaction and Fatigue Version 5 (ProQOL 5) scale and a set of demographic questions developed by the researchers. The demographic questions included information about the ED nurses' education level, years in nursing profession, typical shift length, age, etc. The ProQOL was originally developed by Charles Figley and Beth Hudnall Stamm (1996). The ProQOL version 5 was used to examine the prevalence of $\mathrm{CS}, \mathrm{CF}$, and burnout among ED nurses in this study. The ProQOL 5 is a 30-item self-report scale including three subscales of CS, CF, and burnout. Each subscale has 10 question items and uses a 5-point Likert scale scoring from $1=$ never to $5=$ very often $($ Stamm, 2010).

The ProQOL 5 has good construct validity with over 200 published papers and more than 100,000 internet articles using the scale as a reference and has reported psychometric properties of $\alpha$ reliability ranging from .84 to .90 on the three subscales (Stamm, 2010). The Chronbach's alpha coefficients of internal consistency reliability of the ProQOL 5 for this study were .96 for the total scale, .92 for the CS, .79 for CF subscale, and .82 for the burnout.

\section{Data Analysis}


All of the data were entered into and analyzed by the Statistical Package for the Social Science (SPSS) for Windows, version 21.0. Frequency distributions of all the variables were checked for outliers, missing data, and typing errors. Summary statistics, including the computation of means, standard deviations, frequency counts, and percentages of all demographic and organizational data, were performed according to data levels. Item means, standard deviations, medians, and percentages were computed for the level of CS, CF, and burnout. A series of Pearson $r$ correlation, $t$-test, and one-way analysis of variance (ANOVA) were used to examine the associations between demographics, work-related characteristics, and the level of CS, CF, and burnout. The alpha level was set at .05 for statistical significance. Multiple regression was employed to determine which variables of demographics and workrelated characteristics contributed to the variation of the level of $\mathrm{CS}, \mathrm{CF}$, and burnout. Dummy coding of the work-related characteristic variables was performed when necessary. For using seven selected independent variables to run a multiple regression, this study needed a minimum sample size of 153 subjects to achieve $95 \%$ power and a medium effect size $(.15)$ at alpha $=.05$.

\section{Results}

\section{Demographic Characteristics}

Of the 1,000 surveys mailed to ED nurses nationwide, 284 were returned, representing a $28 \%$ response rate. Because six participants worked less than 8 hours per week, their results were removed from data analysis, leaving the total number of sample at 278. The participants of the study were primarily women $(n=243,87.4 \%)$, white ethnicity $(n=248,89.2 \%)$, and married $(n=190,68.3 \%)$. The mean age was $44(S D=11.47)$ ranging from 24 to 74 years of age. Years working as a nurse ranged from 1 to $48(M=17.58 ; S D=12.67)$. The mean length of years working in the ED was $13.01(S D=9.89)$ ranging from 1 to 40 . The participants' educational 
background varied from diploma $(n=86,30.9 \%)$ to MSN/doctoral degree $(n=55,19.8 \%)$, with the largest number of holding a bachelor's degree $(n=137,49.3 \%)$. Most of the participants worked 12-hour shifts $(n=213,77.2 \%)$.

\section{Prevalence of CS, CF, and Burnout}

Research question one was, "What is the prevalence of CS, CF, and burnout among ED nurses?" Descriptive statistics were used to calculate means, standard deviations, and percentages for $\mathrm{CS}, \mathrm{CF}$, and burnout. The mean scores for the level of CS, CF, and burnout among ED nurses were $39.77(S D=6.32), 21.57(S D=5.44)$, and $23.66(S D=5.87)$, respectively. According to Stamm's (2010) interpretation, 56.8\% of the ED nurses fell into the average level of CS scored from 23 to 41, 65.9\% of the ED nurses were in the low level of CF scored 22 or less, and $54.1 \%$ of the ED nurses were in the average level of burnout scored between 23 and 41 in this study.

\section{Associations between Demographics, CS, CF, and Burnout}

Research question two was, "What demographic characteristics such as age and gender are associated with the prevalence of CS, CF, and burnout among ED nurses?" Pearson $r$ correlation and $t$-test were used to examine the prevalence of $\mathrm{CS}, \mathrm{CF}$, and burnout related to the demographic variables of age and gender. The results showed that the older the nurse was at the time of taking the survey, the higher the level of CS $(r=.260, p=.001)$. The younger the nurse was at the time of taking the survey, the higher the burnout score $(r=-.191, p=.002)$ and the CF ( $r=-.134, p=.027)$. While comparing the difference in the level of CS, CF, and burnout between male and female nurses, there was no statistical significance found.

\section{Associations between Work-Related Characteristics, CS, CF, and Burnout}

Pearson $r$ correlation, $t$-test, and one-way ANOVA were used to answer research question three "What work-related characteristics such as educational level, years in nursing, shift length, 
years worked in the ED, hours worked per week, and having adequate manager support are significantly associated with the prevalence of CS, CF, and burnout among ED nurses?" Scheffe post-hoc comparisons were used to compare if significant differences found in the groups. It was discovered that the CS level for the nurses who held graduate and doctorate degrees was higher than the nurses with the diploma/ADN and the BSN degrees $(F=5.48, p=.005)$. Moreover, those who had masters or doctorate degrees had significantly lower burnout levels than the nurses held the other degree $(F=4.92, p=.008)$. No significant differences in CF between educational backgrounds were identified in this study.

The relationship between years as a nurse, years as a nurse working in the ED, average hours worked per week, and the level of CS, CF, and burnout was computed using Pearson's bivariate correlations, respectively. The result indicated that the more years a nurse has practiced the higher level of CS $(r=.269, p=.001)$ and the lower level of burnout $(r=-.182, p=.003)$. There was no statistically significant relationship between years a nurse has practiced and the CF level.

Additionally, the more years a nurse worked in the ED a higher level of CS ( $r=.264, p=.001)$ and a lower level of burnout $(r=-.183, p=.003)$ they had. There was no significant relationship between years a nurse worked in the ED and the level of CF. Also, no significant relationships between average hours ED nurses worked per week and the level of CS, CF, and burnout were identified.

While comparing the difference in the level of $\mathrm{CS}, \mathrm{CF}$, and burnout between length of shifts and the support of managers respectively, $t$-tests were computed to find that nurses worked for the 8to 10-hour shift produced a higher level of CS $(t=2.47, p=.014)$ and a lower level of burnout $(t=-$ $3.34, p=.001)$ than nurses worked for the 12-hour and 'other' shift, respectively. No significant difference in CF between nurses worked for the 8 to 10-hour shift and the 12-hour and other shift was found. Regarding the support receiving from the manager, it is noted that nurses perceived 
receiving support from the manager had a higher level of CS $(t=3.99, p=.001)$ and a lower level of $\mathrm{CF}(t=-2.89, p=.005)$ and burnout $(t=-5.64, p=.001)$.

\section{Factors for Predicting the Level of CS, CF, and Burnout}

In order to identify which significant variables of demographics and work-related characteristics can predict the level of $\mathrm{CS}, \mathrm{CF}$, and burnout, multiple regression was employed for research question 4. Seven significant variables of demographics and work-related characteristics identified from research questions 2 and 3 were entered into the regression equation using the stepwise solution. As shown in Table 1 , age $(\beta=.239, p<.01)$ and manager support $(\beta=.292, p<.01)$ significantly and positively predicted the level of CS, whereas only manager support $(\beta=-.230, p<.01)$ significantly and negatively predicted the level of $\mathrm{CF}$. In addition, age $(\beta=-.166, p<.05)$ and manager support $(\beta=-.373, p<.01)$ significantly and negatively predicted the level of burnout. Apparently, manager support was the major predictor contributed to the level of CS $\left(8.5 \%\right.$, adjusted $\left.R^{2}=.122, F=17.36, p<.01\right), \mathrm{CF}(5.3 \%$, adjusted $\left.R^{2}=.055, F=7.76, p<.01\right)$, and burnout $\left(13.8 \%\right.$, adjusted $\left.R^{2}=.148, F=21.26, p<.01\right)$.

\section{Discussion}

\section{Level of CS, CF, and Burnout}

In this study, the results indicated a low to average level of CF and burnout among the ED nurses, which is not consistent with the results of the two previous studies (Dominguez-Gomez \& Rutledge, 2009; Hooper et al., 2010) related to ED nurses who perceived significantly higher levels of these two negative aspects. Due to this study's participants being members of the ED professional organization, perhaps they were more involved and invested in their careers than the non-ENA counterparts.

Compassion satisfaction occurs when the care provider feels a sense of connection with his/her patients and feels a sense of achievement in his/her work (Stamm et al., 2010). The positive 
aspect of caring for others and providing support for those in need may outweigh the difficulties of the job. Although the CS level among ED nurse was average in this study, the possible reason might be this group's nurses were more senior and encompassed a more confident outlook of compassion satisfaction toward the positive aspects of nursing. Low levels of CS are a known factor in nursing turnover in the ED (Sawatzky \& Enns, 2012). Not only should the nursing profession pursue the likely causes to $\mathrm{CF}$, but it must further investigate the factors that contribute to CS in ED nurses.

\section{Demographic-Related Characteristics and CS, CF, and Burnout}

Compassion fatigue is less prevalent with increasing age and working experience (Hill \& Stephens, 2003). Correspondingly, this current study demonstrated that older nurses had higher CS scores, as well as lower CF and burnout levels. Specific challenges are present for new, younger nurses. Not only are they inexperienced and challenged to learn new information daily, but must also maintain stride in a busy work environment where speed and skill are critical. The ED leadership and experienced senior nurses must provide a supportive and collaborative environment for newer nurses. Perhaps a formal mentoring program would be helpful to pair a new ED nurse with a more established nurse.

\section{Work-Related Characteristics and CS, CF, and Burnout}

Crucial factors which surfaced in this study as significant elements in ED nurses who exhibited higher CS levels and lower burnout levels included increased years in the profession, more years in the ED, a higher level of educational background, a shorter shift length, and adequate manager support at work. The above mentioned findings are consistent with previous research in which the influence of a positive work environment and more working experience leads to more satisfied nurses (Friedrich, Prasun, Henderson, \& Taft, 2011; Hoar, 2011; Torangeau, Cummings, Cranley, Ferron, \& Harvey, 2010; Li, Early, Mahrer, Klaristenfeld, \& 
Gold, 2014). The more attentive and involved ED managers are, the higher the CS scores of their nurses. Healthy, happy work environments which include manager support, shared decisionmaking, and recognizing nurses' contributions to practice are precisely associated with increased nurse retention, reduced staff turnover, and increased job satisfaction (American Organization of Nurse Executives, 2003; Leiter \& Laschinger, 2006).

\section{Factors for Predicting the Level of CS, CF, and Burnout}

This study identified specific demographic and work-related characteristics which influence a nurse's level of happiness and satisfaction, as well as CF and burnout at work. A critical modifiable feature related to predict the level of $\mathrm{CS}, \mathrm{CF}$, and burnout, was manager support. While influences such as age are not changeable, the nursing leaders might acknowledge the younger nurses may be at risk for developing burnout and $\mathrm{CF}$ at work.

A key concern is that EDs are becoming increasingly busier and more stressful. According to the Center for Disease Control and Prevention (CDC, 2010), ED visits in the U.S. have increased 37\% from 1995 to 2010, hitting a new high of over 124 million visits in 2010. In addition, the CDC expects ED volumes to increase 5\% annually for the next five years. Certainly, these statistics are going to make an ED nurse's job more challengeable. The prevalence of $\mathrm{CF}$ and burnout will most likely continue to grow unless further strategies and solutions are made available to decrease the severity. Compassion fatigue and burnout may have severe professional consequences such as affecting the ability to care for others (Wisniewski, 2011; Sabo, 2011; Boyle, 2011) and affecting nurse retention, patient safety, and patient satisfaction (Burtson \& Stichler, 2010; Hooper et al., 2010; Potter et al., 2010).

A positive, supportive manager is more likely to have nurses who have high levels of CS, as well as lower levels of burnout. Nurse leaders must become cognizant of nurses who are at higher risk for $\mathrm{CF}$ and burnout and have a positive relationship with them in order to 
appropriately counsel and communicate with them. These leaders are crucial in the successful development of strong, positive, professional practice environments (Laposa, Alden, \& Fullerton, 2003). By building a supportive environment, perhaps the early recognition of CF and burnout in ED nurses and providing adequate manager support may aid in the retention of knowledgeable, caring, experienced nurses.

\section{Limitations and Recommendations}

A small sample size with a low response rate was a limitation for this study. Although 1,000 surveys were mailed to the ENA members, only 284 were returned. The results might not be generalized to all ED nurses and who were not ENA members. The prevalence of CS, CF, and burnout were measured at a single point in time and it is possible that an individual's assessment of his or her perceptions changes over time due to individual work-related conditions (Stamm, 2010). Moreover, ED nurses' perceptions of CS, CF, and burnout are subjective, and their perceptions may be affected by variables that were not examined in this study.

Further research could lead to the development of programs that help ED nurses manage the strain of caring for difficult patients. Additional exploration may be directed toward examining coping strategies that may prevent the development of $\mathrm{CF}$ and burnout. Future research concentrating on a more detailed view of the finding that older and more experienced nurses had higher levels of CS would be very beneficial for the nursing profession. It may be possible that more experienced nurses could be the key in assisting newer, younger nurses to find strategies that can improve their quality of life at work and perhaps preventing burnout and CF.

\section{Conclusion}

Overall results of this study revealed average to low levels of CF and burnout and average to high levels of CS among this group of ED nurses. The demographic and work-related characteristics such as age, educational background, and years as a nurse influenced the prevalence of CS, CF, and 
burnout among ED nurses. A key predictor, manager support, predicted the CS, CF, and burnout in this study. An increased awareness of CF and burnout may aid in improved ED nurse job satisfaction, and therefore, increased quality patient care. It is imperative that the nursing profession addresses support, strategies, and solutions that may facilitate a higher level of work satisfaction among ED nurses.

\section{Clinical Resources:}

www.proqol.org

Professional quality of life information, including compassion fatigue/burnout

Professional Quality of Life Scale self-test

www.compassionfatigue.org

Information for caregivers

www.giftfromwithin.org

Information and articles for post-traumatic stress syndrome survivors and their caregivers

\section{References}

American Organization of Nurse Executives (2003). Healthy work environments, volume II: Striving for excellence. Retrieved from http://www.aone.org/aone/keyissues/hwe_excellence.html

Boyle, D. A. (2011). Countering compassion fatigue: A requisite nursing agenda. Online Journal of Issues in Nursing, 16(1), 1-16.

Burtson, P. L., \& Stichler, J. F. (2010). Nursing work environment and nurse caring: relationship among motivational factors. Journal of Advanced Nursing, 1819-1831.

Centers for Disease Control and Prevention (2010). Retrieved from http://www.cdc.gov/nchs/ahcd.htm

Dominguez-Gomez, E., \& Rutledge, D. N. (2009). Prevalence of secondary traumatic stress among emergency nurses. Journal of Emergency Nursing, 35(3), 199-204.

Figley, C. R. (1995). Compassion Fatigue: Coping With Secondary Traumatic stress disorder in those who treat the traumatized. New York, NY: Brunner/Mazel. 
Figley, C. R. (2002). Compassion fatigue: Psychotherapists' chronic lack of self care. Journal of Clinical Psychology, 58(11), 1433-1441.

Figley, C.R. \& Stamm, B.H. (1996). Psychometric review of compassion fatigue self test. In: B.H. Stamm (Ed.), Measurement of stress, trauma, and adaptation. Lutherville, MD. Sidran Press.

Flarity, K. (2011). Compassion fatigue. ENA Connection, 35(7), 10.

Flarity, K., Gentry J. E., Mesnikoff, N. (2013). The effectiveness of an educational program on preventing and treating compassion fatigue in emergency nurses. Advanced Emergency Nursing Journal, 35 (3), 247-258.

Friedrich, L.A., Prasun, M.A., Henderson, L., \& Taft, L. (2011). Being a seasoned nurse in active practice. Journal of Nursing Management. 19(7), 897-905.

Healy, S., \& Tyrrell, M. (2011). Stress in emergency departments: Experiences of nurses and doctors. Emergency Nurse, 19(4), 31-37.

Hill, R. P., \& Stephens, D. L. (2003). The compassionate organization in the 21 st century. Organizational Dynamics, 32(4), 331-341.

Hoar, P. A. (2011). Nurse manager emotional intelligence, staff nurse satisfaction, and staff nurse retention. (Order No. 3484366, Teachers College, Columbia University). ProQuest Dissertations and Theses, 255. Retrieved from http://search.proquest.com/docview/903918923? accountid=4488. (903918923).

Hooper, C., Craig, J., Janvrin, D. R., Wetsel, M. A., \& Reimels, E. (2010). Compassion satisfaction, burnout, and compassion fatigue among emergency nurses compared with nurses in other selected inpatient specialties. Journal of Emergency Nursing, 36(5), 420427. 
Laposa, J. M., Alden, L. E., \& Fullerton, L. M. (2003). Work stress and posttraumatic stress disorder in ED nurses/personnel. Journal of Emergency Nursing, 29(1), 23-28.

Leiter, M. P., \& Laschinger, H. K. (2006). Relationships of work and practice environment to professional burnout. Nursing Research, 55(2), 137-146.

Li, A., Early, S.F., Mahrer, N.E., Klaristenfeld, J.L., \& Gold J.I. (2014). Group cohesion and organizational commitment: protective factors for nurse residents' job satisfaction, compassion fatigue, compassion satisfaction, and burnout. Journal of Professional nursing, 30(1), 89-99.

McHugh, M. D., Kutney-Lee, A., Cimiotti, J. P., Sloane, D. M., \& Aiken, L. H. (2011). Nurses' widespread job dissatisfaction, burnout, and frustration with health benefits signal problems for patient care. Health Affairs, 30(2), 202-210.

Medicare (2013). Medicare.gov the official U.S. government site for Medicare. Retrieved from http://medicare.gov

Portnoy, D. (July-August, 2011). Burnout and compassion fatigue: Watch for the signs. Journal of the Catholic Health Association of the United States Health Progress, 46-50. Retrieved from www.chausa.org

Potter, C. (2006). To what extent do nurses and physicians working within the emergency department experience burnout? A review of the literature. Australasian Emergency Nursing Journal, 9(2), 57-64.

Potter, P., Divanbeigi, J., Berger, J., Cipriano, D., Norris, L., \& Olsen, S. (2010). Compassion fatigue and burnout: Prevalence among oncology nurses. Clinical Journal of Oncology Nursing, 14(5), E56-E63.

Sabo, B. (2011). Reflecting on the concept of compassion fatigue. Online Journal of Issues in Nursing, 16(1), 1-22. 
Sawatzky, J. V., \& Enns, C. L. (2012). Exploring the key predictors of retention in emergency nurses. Journal of Nursing Management, 20, 696-707.

Stamm, B. H. (2010). The Concise ProQOL Manual (2ed ed.). Pocatello, ID. Retrieved from http://www.ProQOL.org

Stamm, B. H., Blampied, S., Higson-Smith, C., Hudnall, A. C., Piland, N. F., Stamm, H. E., \& Khabir, S. (2010). ProQol.org. Retrieved from http://www.proqol.org

Torangeau, A., Cummings G., Cranley L., Ferron E., \& Harvey, S. (2010). Determinants of hospital nurse intention to remain employed: broadening our understanding. Journal of Advanced Nursing, 66(1), 22-32.

Vahey, D. C., Aiken, L. H., Sloane, D. M., Clarke, S. P., \& Vargas, D. (2004). Nurse burnout and patient satisfaction. Med Care, 42(2), 1157-1166.

Von Rueden, K. T., Hinderer, K., Murray, M., Logan, T., Kramer, B., Gilmore, R., \& Friedmann, E. (2010). Secondary traumatic stress in trauma nurses: Prevalence and exposure, coping, and personal/environmental characteristics. Journal of Trauma Nursing, 17(4), 191-200.

Wisniewski, L. (2011). Is it stress, burnout, or compassion fatigue? Retrieved from http://www.nursetogether.com/Career/Career-Article/itemID/2652/Nurses-is-it-stressburnout-or-compassion-fatig.aspx 